\title{
CEREBRAL GLUCOSE METABOLISM MEASURED BY POSITRON EMISSION TOMOGRAPHY IN PRETERM, TERM NEWBORN INFANTS AND NEONATAL HYPOXIC- ISCHEMIC ENCEPHALOPATHY
}

\author{
Y.Shi ${ }^{1}$, R. Jin ${ }^{2}, \mathrm{H} . \mathrm{Li}^{1}$ \\ ${ }^{1}$ Pediatrics, ${ }^{2}$ Nuclear Medicine, Daping Hospital, Third Military Medical University, Chongqing, China
}

Objective: To study the clinical value of cerebral glucose metabolism measure by ${ }^{18} \mathrm{~F}$-fluorodeoxyglucose ${ }^{18}$ F-FDG PET in preterm, term newborn infants and neonatal hypoxic-ischemic encephalopathy (HIE).

Methods: To measure the cerebral glucose metabolism by ${ }^{18}$ F-FDG PET imaging in newborn infants. Sixty infants were included in the study. The data was collected by PET instrument after administration of $0.1 \mathrm{mCi}$ $/ \mathrm{kg}{ }^{18} \mathrm{~F}$-FDG, and standardized uptake values (SUV) were calculated to estimate the cerebral glucose metabolism.

Results: The cerebral glucose metabolism was found to have a trend to be increased, and the structure of brain ${ }^{18} \mathrm{~F}$-FDG PET imaging to be clear with the increase of gestational age, especially when the gestational age was older than $37 \mathrm{wk}$. The brain ${ }^{18} \mathrm{~F}$-FDG PET imaging in different gestational age showed that the uptake of ${ }^{18} \mathrm{~F}$-FDG was relatively higher in thalamus, cerebellum, sensorimotor cortex and basal ganglia, where as relatively lower in cerebral cortex. The uptakes of ${ }^{18} \mathrm{~F}-\mathrm{FDG}$ in $\geq 37 \mathrm{wk}$ group were found to be significantly higher than those in $\leq 32 \mathrm{wk}$ group $(p<0.01)$. The cerebral glucose metabolism was significantly changed in neonatal HIE. The cerebral uptake of ${ }^{18} \mathrm{~F}-\mathrm{FDG}$ was either unbalanced bilaterally or relatively low in all sites especially in subcortical alba, thalamus, basal ganglia in HIE patients. Moreover, The uptakes of ${ }^{18}$ F-FDG in severe HIE patients were found to be significantly lower than those in mild and medium HIE patients $(\mathrm{p}<0.05)$.

Conclusions: Cerebral glucose metabolism measured by ${ }^{18}$ F-FDG PET might be a useful tool for estimating the brain development and injury in newborn infants, and its clinical values need further investigating. 